\title{
A direct gas chromatography-mass spectrometry (GC-MS) method for the detection of orellanine present in stomach content (Part I)
}

\author{
Ilia Brondz ${ }^{1^{*}}$, Eviatar Nevo ${ }^{2}$, Solomon P. Wasser ${ }^{3}$, Anton Brondz ${ }^{4}$ \\ ${ }^{1}$ Department of Biology, University of Oslo, Oslo, Norway; ${ }^{*}$ Corresponding Author: ilia.brondz@bio.uio.no \\ ${ }^{2}$ International Graduate Center of Evolution University of Haifa, Haifa, Israel \\ ${ }^{3}$ Institute of Evolution, Haifa University, Haifa, Israel \\ ${ }^{4}$ Department of Chemistry, Norwegian University of Science and Technology, Trondheim, Norway
}

Received 29 October 2011; revised 5 December 2011; accepted 20 December 2011

\begin{abstract}
Intoxication with Cortinarius orellanus Fr. is often lethal or, at the least, disabling for the victim. Orellanine is recognized as the prime toxic substance in this mushroom. Poisoning by other toxic mushrooms can be occasionally mistaken as poisoning by $C$. orellanus or vice versa. The C. orellanus toxins have a prolonged latent period after ingestion and onset in the appearance of symptoms. These properties of the toxin together with the chemo-, thermo- and photolability have made it difficult to develop a direct analytical method for diagnosing poisoning with orellanine, which in turn is needed to administer the correct medication. The aim of this study was to develop a direct analytical method for the detection of orellanine present in stomach content. Gas chromatography-mass spectrometry with supersonic molecular beams (GC-MS with SMB) was used for the direct detection of orellanine in the stomach fluids of rats after they were fed with food containing $C$. orellanus. This method can be used as a platform for the future development of analytical procedures for the direct analytical detection of orellanine in humans intoxicated by ingestion of toxic mushrooms. The standard orellanine was isolated from $C$. orellanus following the procedure described by Prast et al. [1] and was used as an authentic comparison.
\end{abstract}

Keywords: Cortinarius orellanus Fr.; Orellanine; GC-MS with SMB; Nephrotoxicity; Nephrotoxin

\section{INTRODUCTION}

The Cortinariaceae are a large family of gilled mush- rooms found worldwide, containing over 2100 species. The deadly toxin orellanine has been found in at least 34 Cortinariaceae. Cortinarius orellanus Fr. inhabit also Northern Europe and stretch as far north as Southern Scandinavia. Poisoning by C. orellanus and other orellanine-containing mushrooms is quite common in East-, North- and Central Europe and North America. Cases of intoxication from orellanine-containing mushrooms have also been registered in Asia.

C. orellanus has attracted the attention of mycologists, chemists, toxicologists and physicians as a mushroom that causes intoxication of humans after ingestion. After an incident of mass intoxication in Poland in the 1950s, the substance orellanine was recognized as the prime toxic substance in this mushroom. Orellanine is recognized as a potent nephrotoxin. Besides orellanine, $C$. orellanus contains several other toxins such as cortinarin $\mathrm{A}, \mathrm{B}$ and $\mathrm{C}$ and degradation products formed from orellanine such as orelline.

The molecular weight of orellanine is 252.039 , the molecular formula is $\mathrm{C}_{10} \mathrm{H}_{8} \mathrm{O}_{6} \mathrm{~N}_{2}$, its systematic name is 3 , 3',4,4'-tetrahydroxy-2,2'-bipyridyl-N,N'-dioxide and the structure is shown in Figure 1(a). The molecular weight of orelline is 220.048 , the molecular formula is $\mathrm{C}_{10} \mathrm{H}_{8} \mathrm{O}_{4} \mathrm{~N}_{2}$, its systematic name is 3,3',4,4'-tetrahydroxy-2,2'-bipyridyl and the structure is shown in Figure 1(b).

The kidneys are the prime target for orellanine intoxication. Despite orellanine being widely recognized as the prime nephrotoxin in C. orellanus [1], some scientists express doubt about the correct correlation between the molecular structure of orellanine and its toxicity [2]. The existence of diglucoside with orellanine as the aglycon has been discussed in the literature and the exact structure of the nephrotoxin in C. orellanus may also be questioned.

There could be a delay (latent period) of up to three weeks from the time of ingestion of food containing $C$. 


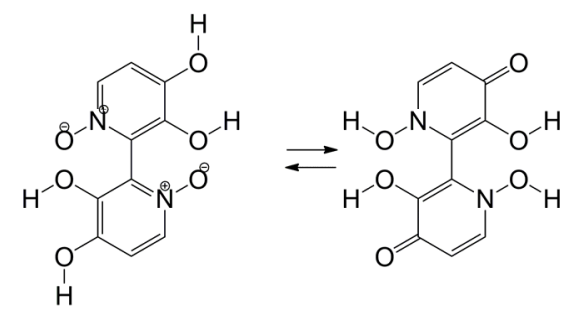

(a)<smiles>CCOc1ccnc(-c2nccc(O)c2O)c1O</smiles>

(b)

Figure 1. (a) The chemical structures for orellanine; (b) The chemical structures for orelline.

orellanus until the onset of symptoms of intoxication. Orellanine intoxication in humans is characterized by a latent period from three days to several weeks before the appearance of poisoning symptoms such as acute renal failure resulting from damage to the tubular epithelium. The term "paraphalloid syndrome" $[3,4]$ is used to describe intoxication, which is associated with a prolonged latent period between ingestion and the appearance of symptoms. The mushrooms that cause symptoms more than $6 \mathrm{~h}$ after consumption are associated with serious and potentially lethal toxicity. C. orellanus is one of these and the precise diagnosis of orellanine as the prime toxin in $C$. orellanus is urgently needed.

The precise diagnosis of intoxication with $C$. orellanus is needed to administer the correct medication. To confirm poisoning with orellanine, the histology of a kidney biopsy of the victim is currently the diagnostic method for humans.

Thin layer chromatography (TLC) methods for the determination of orellanine have been developed [5-7]; however, most of these methods have been applied to mushroom tissues. Even electron spin resonance spectroscopy (ESR) analysis of the oxidation product from orellanine was described in [6] as a diagnostic tool. The high performance liquid chromatography (HPLC) method has also been described [8]; this method was developed to analyze orellanine directly in the stomach contents of rats after ingestion of C. orellanus. The gas chromatography (GC) method provides both significantly better separation of substances in a mixture than TLC or HPLC and better reproducibility. The gas chromatography-mass spectrometry (GC-MS) analyses are quite sensitive, and extremely small amounts of material can be analyzed.

The logical step forward was the development of GCMS analysis. The use of MS in connection with GC for detection provides a unique capacity to identify unknown substances, or to verify the presence of target molecules in complex mixtures. GC-MS is a core analytical technique with a broad range of applications, including the analysis of pharmaceuticals, pesticides, environmental pollutants, xenobiotics and toxins.

However, conventional GC-MS instrumentation demands the use of elevated temperatures in the injector, column, detector and transfer line to the MS during the separation and detection of substances. The temperature in the injector must be higher than the boiling point of the main substance in the mixture to be analyzed. The conventional capillary columns used in these analyses have a normal column length of $15-30 \mathrm{~m}$. The mobile phase (gas) flow rate is low, at about $1 \mathrm{~mL} / \mathrm{min}$ or less.

Current GC-MS technology suffers from a major limitation in that a relatively small range of volatile, thermally stable compounds are amenable to analysis. The electron impact (EI) ionization mass spectra suffer from the frequent absence of the molecular ion $[\mathrm{M}]^{+}$, and this drawback reduces confidence in sample identification. Less volatile compounds tend to be more fragile and have a higher probability of being thermally labile. Furthermore, to prevent ion-source-induced peak tailing (and contamination) with less volatile compounds, the temperature of the ion source must be increased; this causes a reduction in the relative abundance of the molecular ion for all sample compounds (due to increased sample vibrational energy content). Because of the thermolability of orellanine, the use of conventional GC-MS for its analysis is difficult.

In the past decade, a new type of GC-MS has been developed based on the use of supersonic molecular beams (SMB) (also referred to as "supersonic GC-MS") and its performance capabilities were explored $[9,10]$. SMB are used to interface the gas chromatograph to a mass spectrometer and as a medium for ionization of sample compounds while in the SMB by EI $[9,10]$. In contrast to conventional GC-MS, the temperatures used in GC-MS with SMB are significantly lower, the duration of a single analysis is shorter, the column gas flow is higher and the use of a contact-free fly-through EI ion source technique is applied. The GC-MS with SMB is less damaging for thermolabile substances in comparison to conventional GC-MS [9].

The GC-MS with SMB uses capillary columns with a length of about $4 \mathrm{~m}$ or shorter and the mobile phase (gas) flow can be adjusted up to $8 \mathrm{~mL} / \mathrm{min}$ or higher [9].

In the present paper, the GC-MS with SMB method was developed for the direct analysis of orellanine in the stomach content of rats after ingestion of $C$. orellanus. 
This method can be used as a platform for the future development of analytical procedures for the analysis of orellanine in humans who are suspected to be poisoned by toxic mushrooms. The presence of orellanine in animal stomach fluids was analytically detected by GC-MS with SMB and compared with the mass spectrum of standard orellanine.

The biological samples of stomach content were donated by the R \& D Department, Jupiter Ltd., Norway. The pattern of MS fragmentation for orellanine described in the literature and the MS data recorded by analysis of standard orellanine supported the detection of orellanine in the stomach fluid from laboratory animals.

The use of GC-MS with SMB provided a unique opportunity for orellanine analysis in stomach content after ingestion of meals containing toxic mushrooms because of the preservation of intact orellanine molecules during the analytical procedure.

\section{MATERIALS AND METHODS}

\subsection{Instrumentation and Conditions}

The experimental GC-MS with SMB based on a Varian 1200 GC-MS system is described in detail in [9-11]. The separation of compounds was done with a VF-5HT column, $0.25 \mathrm{~mm}$ I.D., $0.1 \mu \mathrm{m}$ film thickness and $4 \mathrm{~m}$ length (Varian, Middleburg, The Netherlands). The reduction of column length was done in the laboratory. The helium column flow rate was $8 \mathrm{~mL} / \mathrm{min}$.

Samples were dissolved in methanol p.a. quality (Merck, Darmstadt, Germany). $1 \mu \mathrm{L}$ sample at an approximate concentration of $20 \mathrm{ppm}$ was injected with a split ratio of 10:1 using the Varian 1079 injector operated at $200^{\circ} \mathrm{C}$. The GC oven was programmed from $120^{\circ} \mathrm{C}$ to $300^{\circ} \mathrm{C}$ at $30^{\circ} \mathrm{C} / \mathrm{min}$.

Ion source degradation was prevented in view of the use of a contact-free fly-through EI ion source [9-12], and degradation of orellanine in the sample was avoided due to the use of a short column length and a high gas column flow rate $[9,10,13]$. Separation and the detection of stomach content are shown in the reconstructed ion chromatogram (RIC) in Figure 2 and the mass spectrum in Figure 3.

\subsection{Standard}

The mushrooms were collected in vicinity of Oslo, Norway, and freeze-dried to a constant weight. Toxins were extracted from $C$. orellanus as discussed elsewhere. The procedure of extraction and isolation of toxin was as described in [1].

\subsection{Sample Preparation}

Biological samples of stomach content and a short de-

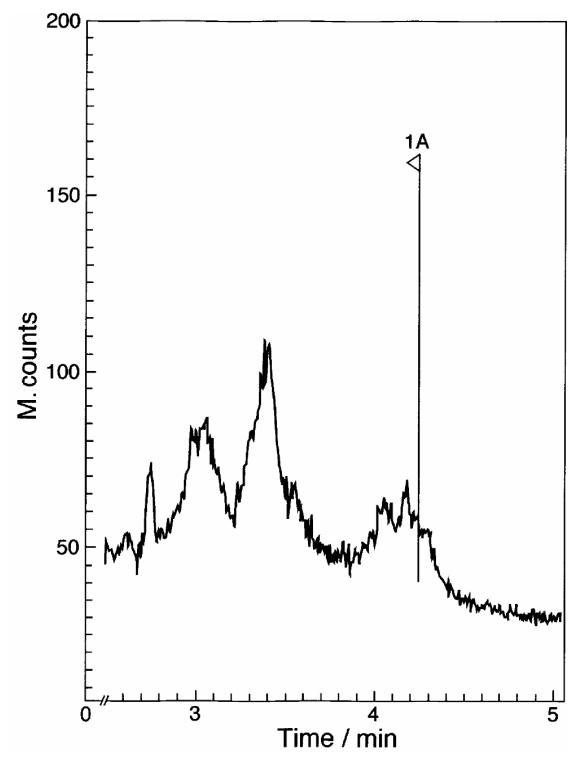

Figure 2. Reconstructed ion chromatogram (RIC) from the stomach content of Mus rattus L. fed with pellets containing dried $C$. orellanus.

scription of the procedure for recovered biological samples were kindly provided by the R \& D Department of Jupiter Ltd., Norway, and were as follows: animals, Musrattus L. were fed with pellets containing dried C. orellanus $(1 \%)$, glucose $(4 \%)$, hydrolyzed protein $(20 \%)$, fat $(10 \%)$ and ground cereals $(65 \%)$, and were given water in quantum satis. After $6 \mathrm{~h}$, the stomach content of the animal was drained by a probe without decapitation of the animals. The obtained stomach content was then lyophilized and samples were kept in liquid nitrogen. Lyophilized stomach content samples $(10 \mathrm{mg})$ were dissolved in methanol (Merck) p.a. quality, homogenized in an ultrasound bath at $0^{\circ} \mathrm{C}$, centrifuged at $10,000 \mathrm{rpm}$ for $15 \mathrm{~min}$, after which the supernatant was separated from the precipitate and lyophilized. Prior to analysis, the samples were adjusted to $0.1 \mathrm{~mL}$ volume with methanol (Merck, Darmstadt, Germany) pro analysis quality.

\section{RESULTS}

Orellanine was detected in the chromatogram by MS as $[\mathrm{M}]^{+}$ions with $\mathrm{m} / \mathrm{z} 252$ as a base signal (Figure 2). The fragmentation of orellanine from the stomach content of an intoxicated rat (Figure 3) was comparable to the spectrum shown in Figure 4, which was published earlier in [8], and to spectra published in the literature $[14,15]$.

The mass spectrum of orellanine showed intense signals at $\mathrm{m} / \mathrm{z} 252[\mathrm{M}]^{+}$, moderate signals at $\mathrm{m} / \mathrm{z} 235$ and weaker signals at $\mathrm{m} / \mathrm{z} 236$ and $\mathrm{m} / \mathrm{z} 220$ in accordance with [14]. The mass spectrum of standard orellanine is shown in Figure 5 and is identical to that for orellanine 


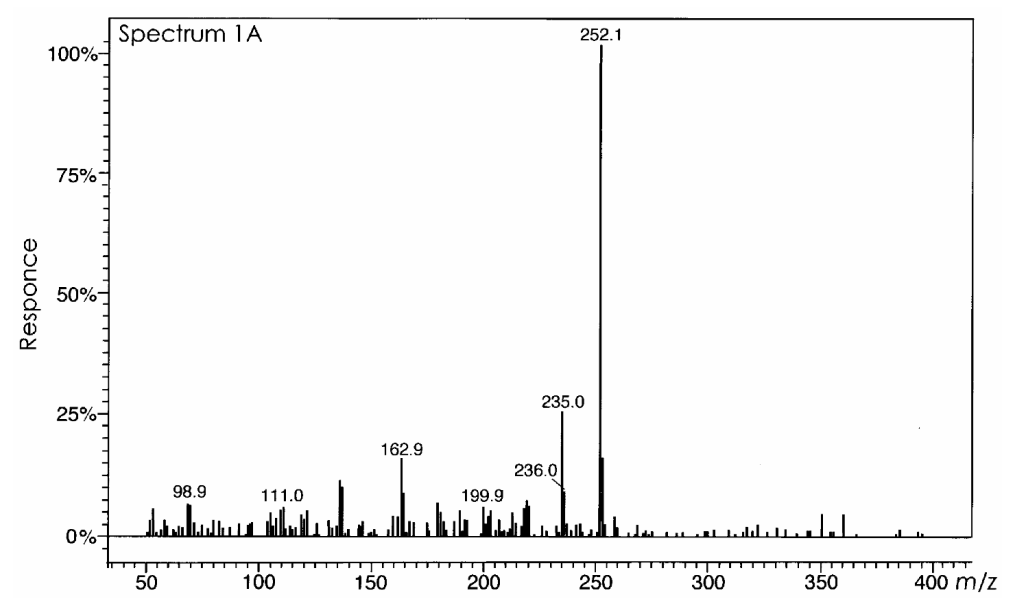

Figure 3. Mass spectrum at point $1 \mathrm{~A}$ in the chromatogram in Figure 2 from the stomach content of Mus rattus L. fed with pellets containing dried $C$. orellanus. The conditions for GC-MS with SMB analyses are described in the text above.

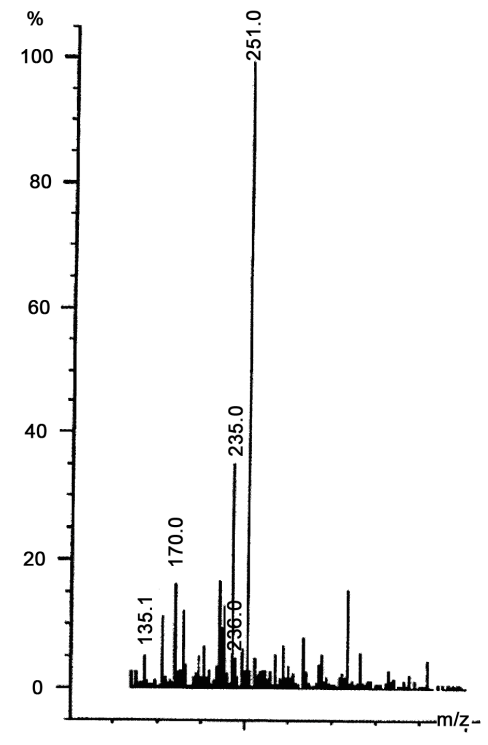

Figure 4. Mass spectrum recorded with negative detection [8].

in biological samples and comparable to previous published in literature $[8,14,15]$.

\section{DISCUSSION}

Several authors in the past and up to present time have studied the toxic properties of $C$. orellanus and the toxin orellanine $[1,4,8,14-20]$. The precise evaluation of cases of human poisoning with orellanine from C. orellanus or other Cortinariaceae requires a kidney biopsy followed by a histological evaluation.

The other possibility is to obtain the stomach content of a victim and to study it by morphological and histological means. However, this procedure often has poor results because the patient usually prepares the meal from several different species of mushroom, which is mixed with several other ingredients and finely cut, cooked or staked, chewed, and until the appearance of the first symptoms, is partly dejected. Because of this, the fine structure of biological material that is mixed together partly disappears.

By the time the initial symptoms appear, severe impairment in renal function is often observed. In a review of Cortinarius species mushroom poisoning, Danel et al. [21] highlighted that from 90 described cases, about $69 \%$ developed symptoms of renal toxicity, more than $51 \%$ of patients required hemodialysis, more than $11 \%$ developed end stage renal failure and more than $13 \%$ required kidney transplantation.

The correct diagnosis and medical treatment is very important in the case of intoxication with C. orellanus and other species containing orellanine. Generally, supportive care for acute kidney injury with close monitoring of serum creatinine is needed [22,23].

The TLC analyses of orellanine in C. orellanus and other toxic mushrooms are described in [24]. TLC is also a useful method for the detection of orellanine in kidney biopsy samples [25]; however, as the authors of this paper concluded: "Orellanine can be detected after a relatively long period following poisoning by performing a simple thin layer chromatography technique using small quantities of renal biopsy material. No toxin was found in urine or blood samples".

Kidney biopsy is an invasive diagnostic method and can be damaging for the patient. In publication [26], the analytical results are presented as a statement: "This report was confirmed by assaying orellanine in the plasma and two renal biopsies of patient after specific photodecomposition into a non-toxic metabolite called orelline". 


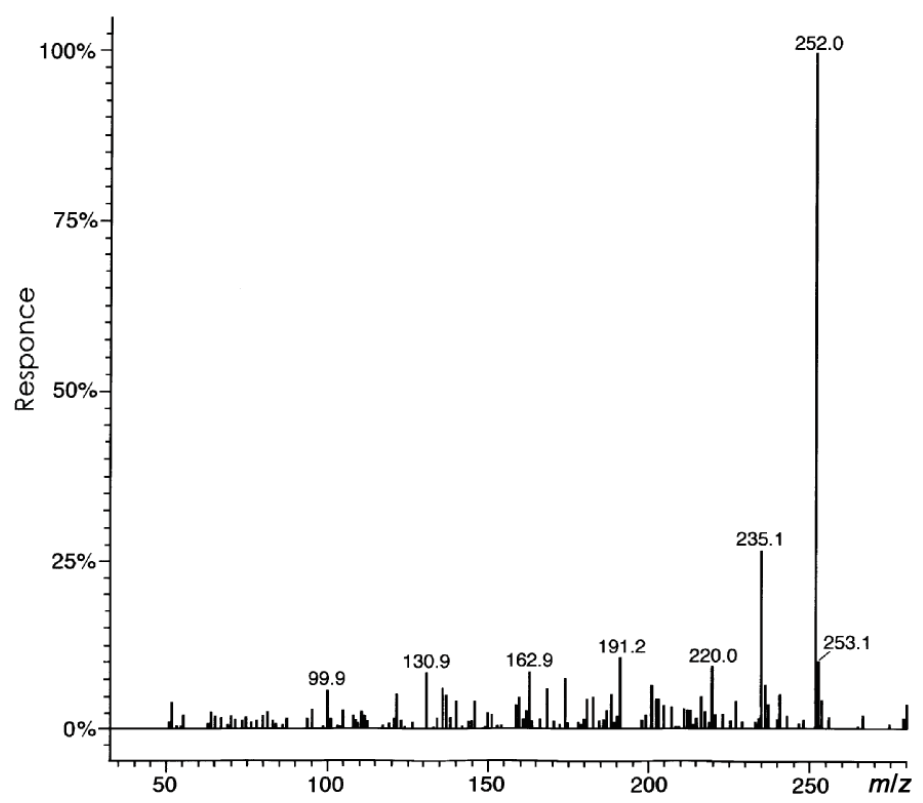

Figure 5. Mass spectrum of the isolated toxin following the method described in [1]. The conditions for GC-MS with SMB analyses are described in the text above.

Orelline is a substance that $C$. orellanus and other toxic Cortinarius species contain as an oxidation/degradation product of orellanine even before ingestion of the mushroom by the victim. The substance can be accumulated in the mushroom under preservation, drying, cooking and staking. It can be accumulated also after ingestion or as a result of sample preparation. Improper sample preparation results in the production of molecules that can mask the nature of the original molecules in the mushroom tissue as described in [27].

The effectiveness of transformation of orellanine to orelline by specific photodecomposition is also not evident [26]. The presence of orelline in mushroom tissues can be detrimental to the accuracy of results. Therefore, the direct determination of orellanine in samples is preferable and needed. In GC-MS with SMB, orellanine was directly detected. The presence of orellanine in samples is supported by the appearance of a well recognizable and distinct base signal of $[\mathrm{M}]^{+}$ions with $\mathrm{m} / \mathrm{z} 252$ in the mass spectrum. Several characteristic fragment ions are also visible in the spectrum. The spectrum is well correlated with earlier published spectra as in Figure 4 [8]. This spectrum is produced by HPLC-MS with negative detection and has a base ion $[\mathrm{M}-\mathrm{H}]^{-}$with $\mathrm{m} / \mathrm{z} 251$. The MS spectrum obtained in the present study is also in good agreement with spectra published in $[1,2,14,15]$.

\section{CONCLUSIONS}

1) GC-MS with SMB gives unequivocal determination of orellanine in biological samples by $[\mathrm{M}]^{+}$ions with $\mathrm{m} / \mathrm{z}$
252 in the mass spectrum and characteristic fragmentation ions.

2) The conditions used during analysis by GC-MS with SMB allow the orellanine molecules to stay intact and gives a visible mass ion signal.

3) The presented method is a basis for direct orellanine analysis. This method can be a useful tool for the determination of the exact cause of intoxication by toxic mushrooms in clinical and forensic medicine.

\section{ACKNOWLEDGEMENTS}

The authors are grateful to Jupiter Ltd., Norway, for donation of biological samples, permission to reproduce mass spectrum recorded with negative detection at HPLC-MS from the stomach content of Mus rattus L. fed with pellets containing dried C. orellanus [8] and financial support. The authors are grateful to Prof. Aviv Amirav, School of Chemistry, Sackler Faculty of Exact Sciences, Tel Aviv University, Tel Aviv, Israel, for technical support and scientific cooperation within MS and to Jon Reierstad, Technical Department, University of Oslo, Norway, for technical assistance in preparation of figures.

\section{REFERENCES}

[1] Prast, H., Werner, E.R., Pfaller, W. and Moser, M. (1988) Toxic properties of the mushroom Cortinarius orellanus. Archives of Toxicology, 62, 81-88. doi:10.1007/BF00316263

[2] Richard, J.-M., Taillandierand, G. and Benoit-Guyod, J.-L. (1985) A quantitative structure-activity relationship study on substituted pyridines as a contribution to the knowledge of the toxic effects of orellanine, a toxin from the 
mushroom Cortinarius orellanus. Toxicon, 23, 815-824. doi:10.1016/0041-0101(85)90013-3

[3] Heim, R. (1963) Diagnoses latines des especes de champignons ou, nonda associes a la folie du komugl tai et du ndaadl (in French). Revue de Mycologie, 28, 277-283.

[4] Schumacher, T. and Høiland, K. (1983) Review mushroom poisoning caused by species of the genus Cortinarius Fries. Archives of Toxicology, 53, 87-106.

[5] Cantin, D., Richard, J.-M. and Alary, J. (1989) Chromatographic behaviour and determination of orellanine, a toxin from the mushroom Cortinarius orellanus. Journal of Chromatography A, 478, 231-237. doi:10.1016/S0021-9673(01)84389-2

[6] Oubrahim, H., Cantin-Esnault, D. and Richard, J.-M. (1996) Novel methods for identification and quantification of the mushroom nephrotoxin orellanine. Part 1: Development of ESR, electrophoresis and TCL methods. Pharmazie, 51, 231-234.

[7] Rapior, S., Andary, C. and Mousain, D. (1987) Cortinarius section orellani isolation and culture of Cortinarius orellanus. Transactions of the British Mycological Society, 89, 41-44. doi:10.1016/S0007-1536(87)80055-4

[8] Brondz, I., Nevo, E., Wasser, S., Høiland, K., Størmer, F. and Brondz, A. (2011) Method development for in vivo analyses of toxin orellanine from the toad mushroom Cortinarius orellanus. Separation Science Europe 2011, London, 10-11 October 2011, Abstract 9.

[9] Brondz, I. (2011) Historical overview of chromatography and related techniques in analysis of antimalarial drug primaquine. Nova Science Publishers, Inc., New York.

[10] Brondz, I., Fialkov, A.B. and Amirav, A. (2009) Analysis of quinocide in unprocessed primaquine diphosphate and primaquine diphosphate tablets using gas chromatography-mass spectrometry with supersonic molecular beams. Journal of Chromatography A, 1216, 824-829. doi:10.1016/j.chroma.2008.11.043

[11] Fialkov, A.B., Steiner, U., Jones, L. and Amirav, A. (2006) A new type of GC-MS with advanced capabilities. International Journal of Mass Spectrometry, 251, 47-58. doi:10.1016/j.ijms.2005.12.045

[12] Amirav, A., Gordin, A., Poliak, M. and Fialkov, A.B. (2008) Gas chromatography-mass spectrometry with supersonic molecular beams. Journal of Mass Spectrometry, 43, 141-163. doi:10.1002/jms. 1380

[13] Fialkov, A.B., Gordin, A. and Amirav, A. (2003) Extending the rage of compounds amenable for gas chromatography mass spectrometry. Journal of Chromatography A, 991, 217-240. doi:10.1016/S0021-9673(03)00247-4

[14] Richard, J.-M. and Ulrich, J. (1989) Mass spectrometry of orellanine, a mushroom toxin, and of related bipyridine$\mathrm{N}$-oxides. Biomedical and Environmental Mass Spectrometry, 18, 1-4. doi:10.1002/bms. 1200180102

[15] Antkowiak, W.Z., Antkowiak, R., Wyrzykiewicz, E. and Czerwiński, G. (1994) Mass spectral fragmentation of orellanine and its tetramethyl ether with regard to their facile thermal and photochemical deoxygenation. Heterocycles, 39, 477-484. doi:10.3987/COM-94-S(B)72
[16] Spiteller, P., Spiteller, M. and Steglich, W. (2003) Occurrence of the fungal toxin orellanine as a diglucoside and investigation of its biosynthesis. Angewandte Chemie International Edition, 42, 2864-2867. doi:10.1002/anie. 200351066

[17] Koller, G.E.B., Høiland, K., Janak, K. and Størmer, F.C. (2002) The presence of orellanine in spores and basidiocarp from Cortinarius orellanus and Cortinarius rubellus. Mycologia, 94, 752-756. doi:10.2307/3761689

[18] Richard, J.-M., Louis, J. and Cantin, D. (1988) Nephrotoxicity of orellanine, a toxin from the mushroom Cortinarius orellanus. Archives of Toxicology, 62, 242-245.

[19] Holmdahl, J., Ahlmén, J., Bergek, S., Lundberg, S. and Persson, S.A. (1987) Isolation and nephrotoxic studies of orellanine from the mushroom Cortinarius speciosissimus. Toxicon, 25, 195-199. doi:10.1016/0041-0101(87)90241-8

[20] Horn, S., Horina, J.H., Krejs, G.J., Holzer, H. and Ratschek, M. (1997) End-stage renal failure from mushroom poisoning with Cortinarius orellanus: Report of four cases and review of the literature. American Journal of Kidney Diseases, 30, 282-286. doi:10.1016/S0272-6386(97)90066-4

[21] Danel, V.C., Saviuc, P.F. and Garon, D. (2001) Main features of Cortinarius spp. poisoning: A literature review. Toxicon, 39, 1053-1060. doi:10.1016/S0041-0101(00)00248-8

[22] Goldfrank, L.R. (2011) Mushrooms. In: Nelson, L.S., Lewin, N.A., Howland, M.A., et al. Eds., Goldfrank's Toxicologic Emergencies, McGraw-Hill, New York.

[23] Brent, J. and Palmer, R.B. (2007) Mushrooms. In: Shannon, M.W., Borron, S.W. and Burns, M.J., Eds., Haddad and Winchester's Clinical Management of Poisoning and Drug Overdose, 4th Edition, Saunders Elsevier, Philadelphia.

[24] Oubrahim, H., Richard, J.-M., Cantin-Esnault, D., SeigleMurandi, F. and Trécourt, F. (1997) Novel methods for identification and quantification of the mushroom nephrotoxin orellanine. Thin-layer chromatography and electrophoresis screening of mushrooms with electron spin resonance determination of the toxin. Journal of Chromatography A, 758, 145-157.

[25] Rohrmoser, M., Kirchmair, M., Feifel, E., Valli, A., Corradini, R., Pohanka, E., Rosenkranz, A. and Pöder, R. (1997) Orellanine poisoning: Rapid detection of the fungal toxin in renal biopsy material. Journal of Toxicology-Clinical Toxicology, 35, 63-66. doi:10.3109/15563659709001167

[26] Rapior, S., Delpech, N., Andary, C. and Huchhard, G. (1989) Intoxication by Cortinarius orellanus: Detection and assay of orellanine in biological fluids and renal biopsies. Mycopathologia, 108, 155-161. doi:10.1007/BF00436220

[27] Brondz, I., Ekeberg, D., Høiland, K., Bell, D.S. and Annino, A.R. (2007) The real nature of the indole alkaloids in Cortinarius infractus: Evaluation of artifact formation through solvent extraction method development. Journal of Chromatography A, 1148, 1-7. doi:10.1016/j.chroma.2007.02.074 